\title{
Unraveling the evolution and coevolution of small regulatory RNAs and coding genes in Listeria
}

Franck Cerutti ${ }^{1}$, Ludovic Mallet ${ }^{1}$, Anaïs Painset ${ }^{1,7}$, Claire Hoede ${ }^{1}$, Annick Moisan ${ }^{1}$, Christophe Bécavin ${ }^{2,3,4,5}$, Mélodie Duval ${ }^{2,3,4}$, Olivier Dussurget ${ }^{2,3,4,6}$, Pascale Cossart ${ }^{2,3,4}$, Christine Gaspin ${ }^{1}$ and Hélène Chiapello ${ }^{1 *}$

\begin{abstract}
Background: Small regulatory RNAs (sRNAs) are widely found in bacteria and play key roles in many important physiological and adaptation processes. Studying their evolution and screening for events of coevolution with other genomic features is a powerful way to better understand their origin and assess a common functional or adaptive relationship between them. However, evolution and coevolution of sRNAs with coding genes have been sparsely investigated in bacterial pathogens.

Results: We designed a robust and generic phylogenomics approach that detects correlated evolution between sRNAs and protein-coding genes using their observed and inferred patterns of presence-absence in a set of annotated genomes. We applied this approach on 79 complete genomes of the Listeria genus and identified fifty-two accessory sRNAs, of which most were present in the Listeria common ancestor and lost during Listeria evolution. We detected significant coevolution between 23 sRNA and 52 coding genes and inferred the Listeria sRNA-coding genes coevolution network. We characterized a main hub of 12 sRNAs that coevolved with genes encoding cell wall proteins and virulence factors. Among them, an sRNA specific to L. monocytogenes species, rli133, coevolved with genes involved either in pathogenicity or in interaction with host cells, possibly acting as a direct negative post-transcriptional regulation.
\end{abstract}

Conclusions: Our approach allowed the identification of candidate sRNAs potentially involved in pathogenicity and host interaction, consistent with recent findings on known pathogenicity actors. We highlight four sRNAs coevolving with seven internalin genes, some of which being important virulence factors in Listeria.

Keywords: Listeria, sRNA, Phylogenomics, Coevolution network, Regulation, Cell wall, Pathogenicity, Internalin

\section{Background}

Small regulatory RNAs are widespread in all kingdoms of life and are recognized as key negative or positive regulators of gene expression [1-3]. They are involved in a wide panel of physiological processes and adaptive responses in bacteria including stress responses, quorum sensing, toxin-antitoxin systems or pathogenicity [4-6]. They generally act post-transcriptionally in cis (antisense) or trans by base pairing with their target messenger RNA (mRNA) but can also bind specific proteins and modify

\footnotetext{
* Correspondence: helene.chiapello@inra.fr

'Université de Toulouse, INRA, UR 875 Unité Mathématiques et Informatique

Appliquées de Toulouse, Auzeville, 31326 Castanet-Tolosan, France

Full list of author information is available at the end of the article
}

their activity, as illustrated by CsrB and 6S sRNA [1]. The most extensively studied class of sRNA includes transencoded sRNAs which regulate their target mRNA by forming short and imperfect duplexes. In silico identification of these duplexes remains a major challenge due to a prohibitively high level of false positive candidates [7-9]. Nevertheless, an improvement in target prediction was shown $[10,11]$ by focusing on site-specific regions such as the ribosome binding site (RBS), the accessibility of unstructured seed regions in both the sRNA and target mRNA, and the use of comparative genomics of interaction candidates. Altogether, these features argue for a better understanding of sRNA history during bacterial evolution and shed light on how regulatory networks 
involving trans-acting-sRNA and target mRNA have emerged and evolved. Unfortunately, little is known about sRNA evolution, sRNA expression control and sRNAmRNA coevolution within bacteria, and very few studies have been carried out on these topics so far. This can be explained by the lack of sRNA annotation in available genome resources as well as by the low number of wellcharacterized regulatory sRNAs and the rapid evolution of regulatory sRNAs in bacteria [1].

In the last decade, high throughput sequencing and transcriptome-wide approaches led to a continuous accumulation of complete genomic data in public databases and contributed to the discovery of hundreds of putative and confirmed new sRNAs in many bacteria such as Escherichia coli [12], Salmonella [13], Bacillus subtilis [14, 15] and Listeria [5, 6, 16-27], giving rise to large-scale comparative analyses and sRNA evolutionary studies. Existing studies on that topic focused on Gramnegative species, including Escherichia coli and related genomes [8, 28-30]. Phyletic analysis of E.coli sRNAs [29] led to the first insights into the distribution of sRNAs in gamma-proteobacteria, greatly improving our understanding of the origin of sRNA-mediated regulation and the underlying mechanisms at the source of sRNA acquisition. To our knowledge, such a global evolutionary study has never been performed in Grampositive bacteria.

Listeria are Gram-positive bacteria that are widespread in the environment and encompass 17 species, two of which are pathogenic: Listeria monocytogenes, the human foodborne agent responsible for listeriosis, and Listeria ivanovii, an animal pathogen [31]. L. monocytogenes has become a model for the study of hostpathogen interactions due to its unique ability to cross host barriers, escape from immune defenses, invade cells and manipulate cellular machineries [32-34]. The comparative analysis of the complete genome sequence of $L$. monocytogenes and the non pathogenic species $L$. innocua in 2001 was the first study to shed light on Listeria virulence and its evolution [35]. Following this pioneer work, Listeria genomic data grew exponentially and more than 80 complete genomes have been sequenced [36]. Small non-coding RNAs were also extensively studied in L. monocytogenes [5, 6, 16-27]. Indeed, 304 non-coding RNAs elements were reported in $L$. monocytogenes EGD-e including 154 sRNAs, 104 antisense and 46 cis-encoded [16, 17, 37]. Among these sRNAs, several were shown to be upregulated in bacteria growing in murine intestinal lumen and in human blood, suggesting that they may play a role in adaptation of the bacteria to the niches occupied during infection $[1,5,21]$.

Comparative analyses of Listeria sRNAs by Kuenne et al. [38] revealed the organization of CRISPR arrays and cas genes in 38 complete L. monocytogenes genomes.
Becavin et al. compared three L. monocytogenes species and observed a high conservation of sRNAs compared to protein-coding genes [37]. A comparative transcriptomics approach was also used to compare the expression of non-coding RNAs in L. monocytogenes and L. innocua species, which revealed conservation across most transcripts, but significant divergence between the species in a subset of non-coding sRNAs [22].

In this article, we present a robust phylogenomics approach that extends and improves existing strategies dedicated to the study of sRNA evolutionary dynamics. We use it to provide the first evolutionary dynamics study of 79 complete genomes of the Listeria genus with regards to protein-coding genes, and a selected set of 112 sRNA loci experimentally identified in the pathogenic strain L.monocytogenes EGD-e. This dataset includes intergenic trans-encoded sRNAs assumed to target independently expressed and distant mRNAs. We built the core and accessory sRNA and coding genes sets and deduced the ancestral presence-absence states for all Listeria genes. Using these patterns, we identified a subset of 23 sRNAs that significantly coevolved with $5^{\prime}$ untranslated regions of coding genes ( $\left.5^{\prime} \mathrm{UTRs}\right)$ and coding DNA sequence (CDS) regions of 52 Listeria coding genes. We reconstructed the coevolution network between sRNAs and coding genes and revealed a hub of 12 sRNAs coevolving with genes encoding cell wall proteins and virulence factors. Among them, we focused on rli133, an sRNA specific of $L$. monocytogenes species that coevolved with 12 coding genes, six of which exhibited a documented function linked to either virulence or interaction with the host cell, possibly acting as a negative post-transcriptional regulator.

\section{Results}

\section{A robust screening strategy for sRNA and coding genes coevolution}

We designed an original approach to build a reference phylogenetic tree to infer observed and ancestral evolution patterns and to identify coevolution relationships between pairs of sRNAs and coding-genes. The four main steps of this approach are presented in Fig. 1 and a full description of each step of the workflow is provided in the Methods section. Briefly, the approach starts from a set of annotated genomes and a list of sRNAs to proceed through four main steps: (1) the construction of a reference phylogenetic tree based on orthologous genes; (2) the construction of the presence-absence matrix for sRNAs, 5'UTRs and CDS parts allowing across all the genomes to define core and accessory sets for all elements; (3) the inference of ancestral presence-absence patterns for all variable sRNAs, 5'UTRs and CDS; and (4) the detection of coevolution events between regulatory sRNA and coding genes and construction of a coevolution 

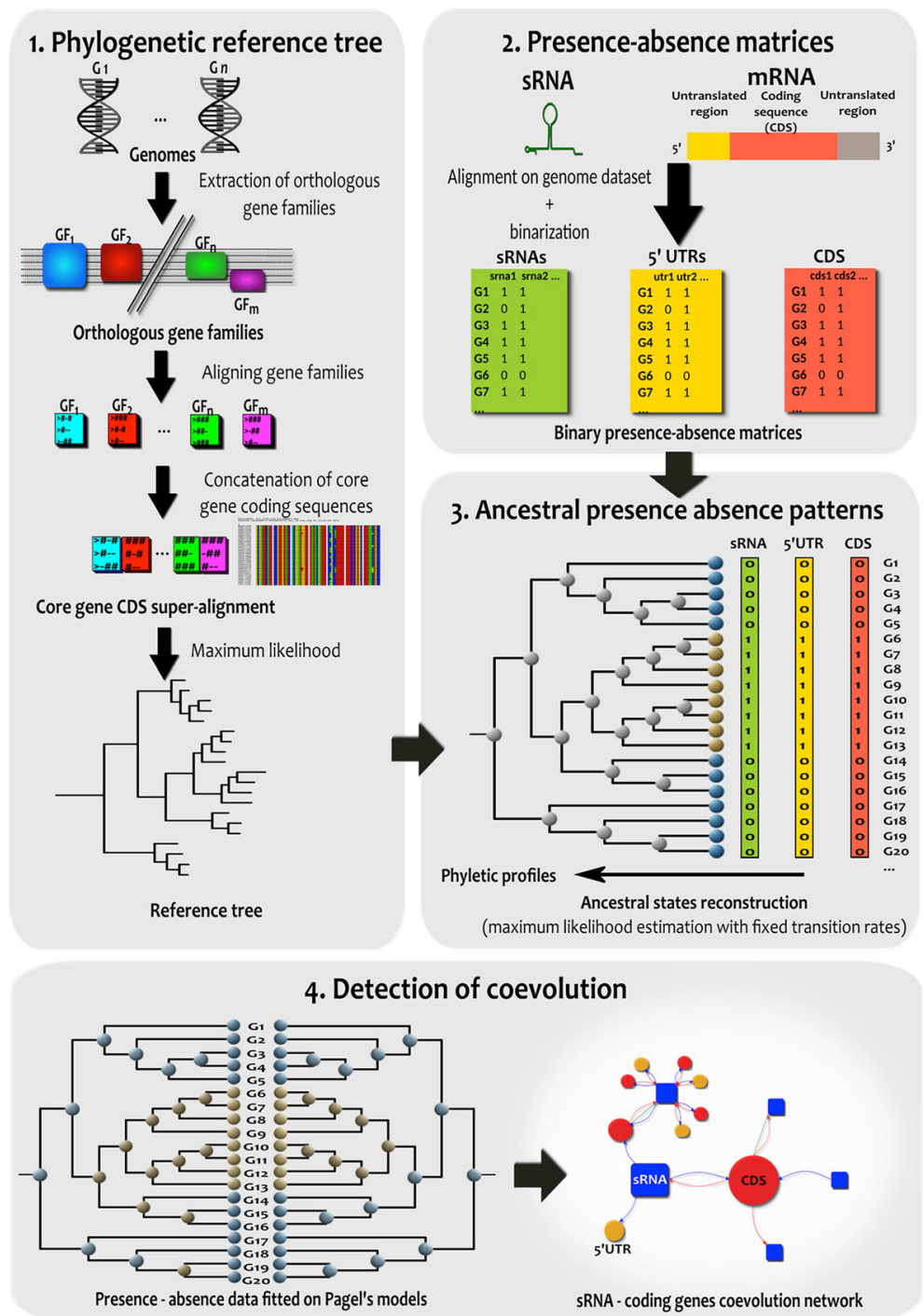

Fig. 1 Strategy and workflow. The strategy consists in 4 steps: (1) Construction of a phylogenetic reference tree computed from a super-alignment of syntenic core genes and a Maximum Likelihood approach (2) Presence-absence matrices computation using alignments of sRNAs, 5'UTRs and CDS (3) Ancestral presence-absence pattern reconstruction for sRNAs, 5'UTRs and CDS based on Markov Model and a Maximum Likelihood approach (4) Detection of coevolution events between sRNAs and 5'UTRs or CDS using both observed and ancestral patterns and construction of the sRNA-coding genes coevolution network

network using both the observed and the reconstructed ancestral presence-absence patterns. The detection of correlated evolution events relies on a phylogenetic-statistical method based on continuous-time Markov modeling of trait evolution developed by M. Pagel [39]. It compares the statistical likelihood of the observed data (in this case, sRNAs, 5'UTRs and CDS presence/absence patterns) under two alternative scenarii: one in which the two features are allowed to evolve independently on the phylogeny, and another where they coevolve together.

This strategy was applied on 112 L. monocytogenes EGD-e putative trans sRNAs, all screened on 79 Listeria genomes (see Additional file 1: Table S1) obtained from the Listeriomics database [36]. To deal with the remaining paralogs in the dataset, sRNAs exhibiting overlapping positions on the EGD-e reference genome were merged in 15 sRNA loci (see Additional file 2: Table S2 and the Methods section for details).

The Listeria phylogenetic reference tree obtained from the 1399 syntenic core coding genes of Listeria was robust and consistent with previous studies [40] (see Fig. 2). The four major phylogenetic lineages of L. monocytogenes were clearly separated with good Shimodaira Hasegawa (SH) supports (Fig. 2b) [40]. We however observed a few branches of lineage I with lower $\mathrm{SH}$ support that correspond to highly conserved genomes, resulting in 


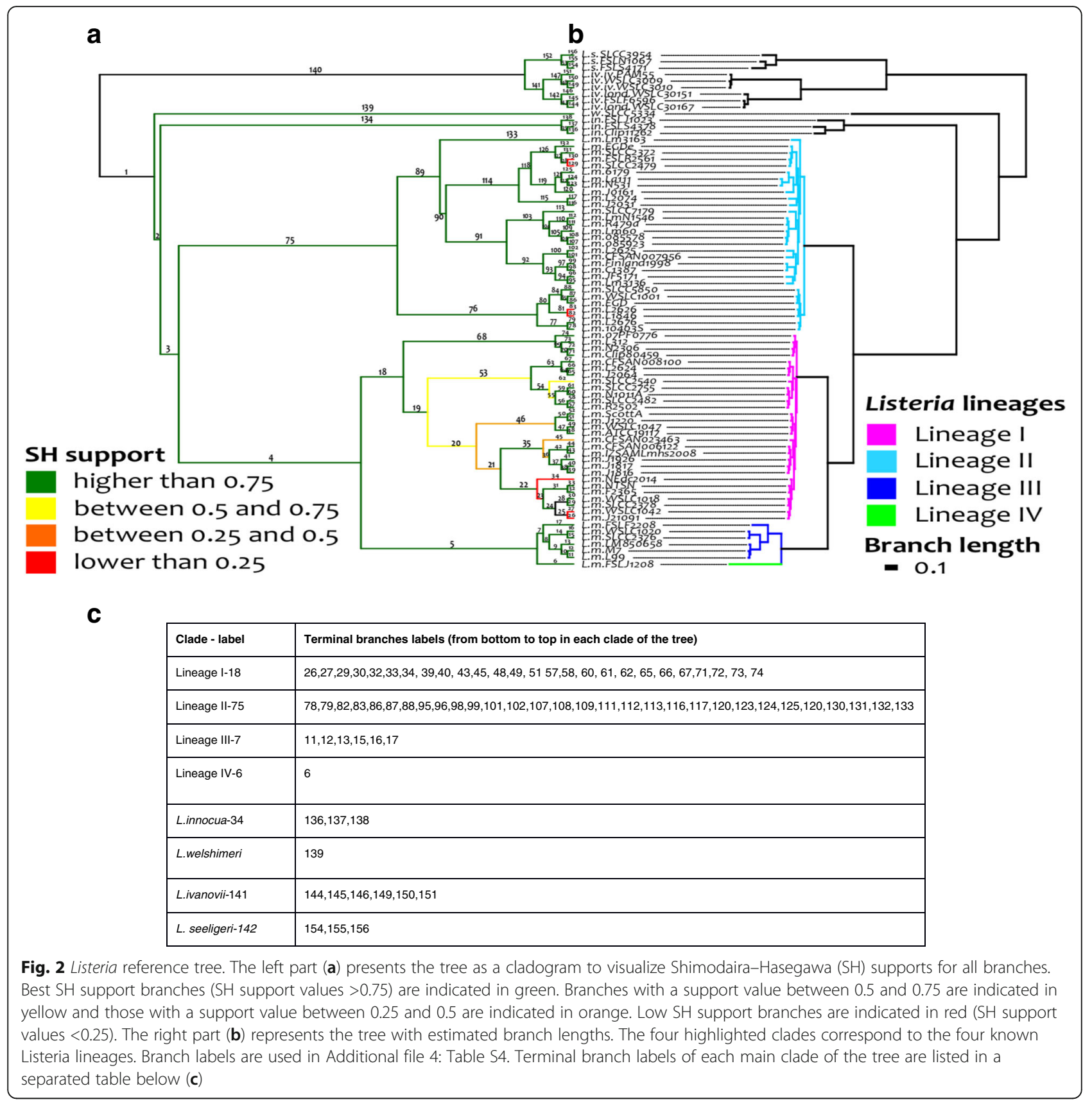

short branch lengths and a weaker phylogenetic signal. This reference tree was subsequently used to compare sRNAs and coding gene content of Listeria genomes.

\section{sRNA content of Listeria genomes}

On the 112 L. monocytogenes EGD-e sRNAs, 52 (46\%) were found to be variable in Listeria genomes, i.e., absent in at least one Listeria genome, and 60 (54\%) were found to be present in all Listeria genomes (Additional file 3: Table S3) the later constitute the core Listeria-sRNAs. Six of these core sRNAs (rli102, rli119, rli120, rli19-ssrA, rli69 and the rli2-LhrC-2_rli4-LhrC-
4_rli7-LhrC-5_rli3-LhrC-3_rli1-LhrC-1 sRNA locus) were kept in the core set despite that their presence could not be confirmed in one or two genomes due to unsequenced regions. Among the core sRNAs, 79\% of their occurrences were located in syntenic regions (meaning that both $5^{\prime}$ and $3^{\prime}$ adjacent genes were also found conserved). We then focused on the 52 variable sRNA loci to decipher their evolutionary history in Listeria genomes.

Small-RNA presence-absence patterns along the Listeria phylogenetic reference tree are shown in Fig. 3. Most sRNAs are present in nearly all genomes, except mostly 


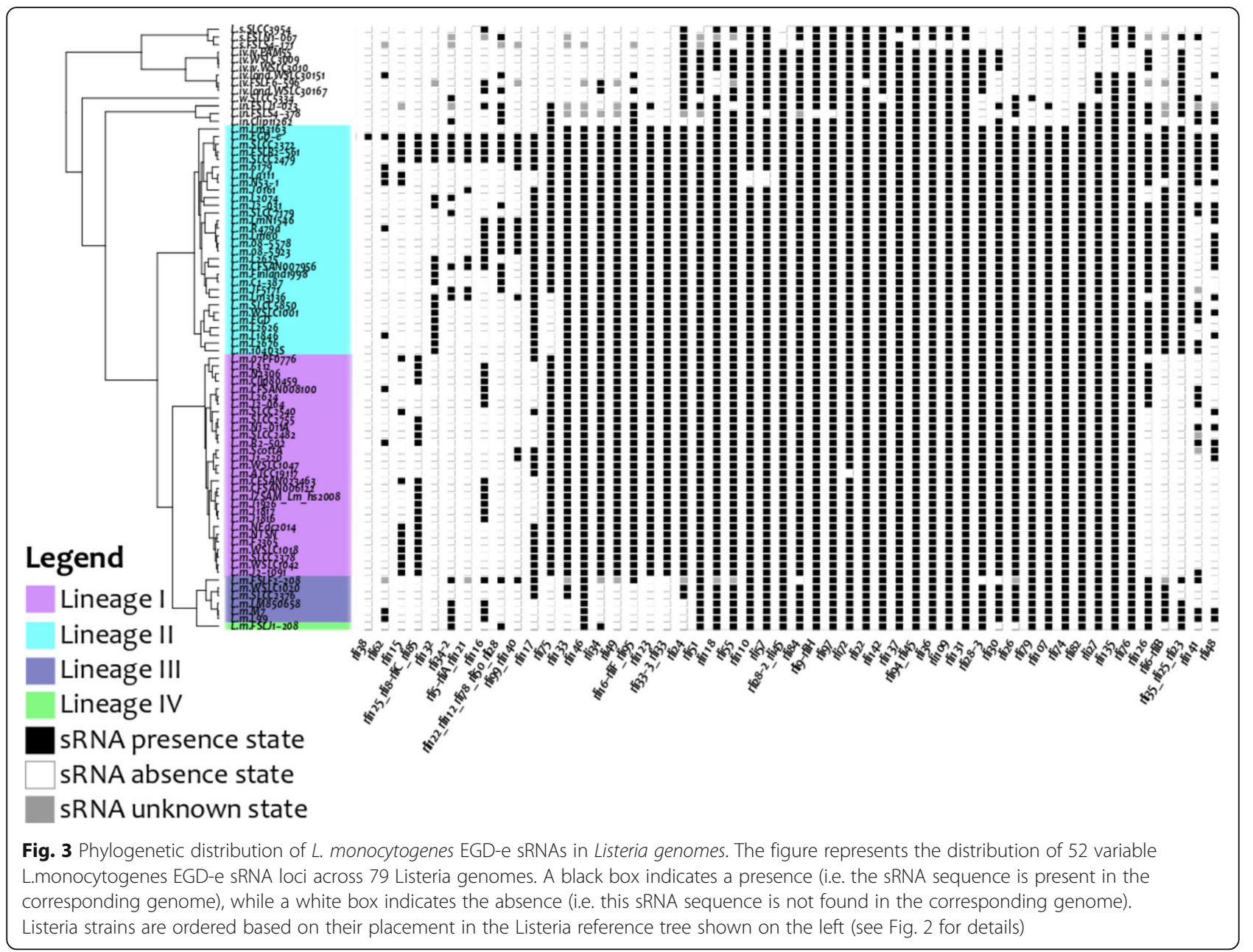

non-L. monocytogenes species. Small-RNA presenceabsence patterns (Fig. 3) also suggest a link between sRNA content and previously defined Listeria lineages. For example most of sRNAs present in genomes of lineage I are also present in genomes of lineages II, but not systematically in lineage III and IV. Two sRNAs are found only in lineages I and II of Listeria monocytogenes (i.e., see rli49 and rli33-3_rli33). Two other sRNAs are systematically absent of lineage I, while they are present in almost all the other Listeria genomes (e.g., rli6-rliB, rli23_rli25_rli35). Rli74 is specifically present in all four $L$. monocytogenes lineages and absent in other Listeria species. Additionally, several sRNAs exhibit sparse presence-absence patterns probably related to complex evolutionary histories.

\section{Listeria SRNA evolution and coevolution profiles}

To investigate sRNA evolutionary histories, we inferred ancestral presence-absence patterns for all 52 variable sRNAs and obtained 44 different phyletic profiles (see Additional file 3: Table S3). Only eight sRNAs shared identical phyletic profiles as following: (1) rli109, rli131, rli36 and rli94_rli45: one loss in branch 154
(L. seeligeri/str. FSL S4-171), (2) rli9-rliH and rli97: one loss in branch 137 (L. innocua/str. FSL S4-378) and (3) rli135 and rli76: one loss in branch 147 (common ancestor of three $L$. ivanovii strains). This indicates that most profiles and evolutionary histories are specific to an sRNA, even if some evolutionary events are shared by several sRNAs. On the basis of the reconstructed sRNA gains and losses on the reference phylogenetic tree, we found only three sRNAs with monophyletic patterns (present in the most-recent common ancestor and all its descendants) (rli146, rli38 and rli74) and two sRNAs with polyphyletic patterns (present in some genomes but not in their most-recent common ancestor) (rli62 and rli99_rli140). All the other 47 (90\%) sRNAs exhibit more or less complex paraphyletic patterns (present in the most-recent common ancestor and some of its descendants). Additionally, several sRNAs have undergone either a large number of gains (e.g., rli116: 10 gains, rli115: 5 gains) or a large number of losses (e.g., rli122_rli112_rli78_rli94_rli50_rli28 locus: 13 losses; rli141: 10 losses; rli26, rli48 and rli117: 8 losses) or both (e.g., rli48: 8 gains and 8 losses), 
suggesting that some sRNAs are subject to frequent reshuffle, even at short evolutionary scales.

The three monophyletic profiles indicate a scenario of gene appearance and descent. For instance, rli146 and rli74 exhibit the same monophyletic profile, i.e., one acquisition at branch 3 (ancestor of L. monocytogenes strains). It was inferred that rli38 was acquired at branch 132 in L. monocytogenes EGD-e. Two different and complex polyphyletic patterns observed for Rli62 and rli99_rli140 suggest potential Horizontal Transfer events. All other sRNAs exhibit paraphyletic patterns, suggesting they underwent one or several loss events in the Listeria reference tree. Thirty-five out of 47 sRNAs (74\%) with paraphyletic patterns are inferred to be present at the tree root, suggesting that the majority of sRNAs were present early, in Listeria evolution.

\section{The Listeria sRNA-coding gene coevolution network}

We used Pagel's model statistical framework [39] (see Methods) and both observed and ancestral presence/absence states to identify significant coevolution relationships between sRNAs and 5'UTRs/CDS regions along the Listeria reference tree. We obtained 23 putative sRNAs showing significant coevolutionary relationships with 23 5'UTRs and 39 CDS of 52 coding genes (see complete list in Additional file 4: Table S4).

All results of sRNAs, 5'UTRs and CDS phyletic patterns, coevolution analyses and the resulting coevolution network were made available on a dedicated web server that provides several facilities to browse the results: http://genoweb.toulouse.inra.fr/Listeria_sRNA. The web application was developed with the Shiny technology [41] and allows interactive visualization of individual phyletic patterns (i.e., observed and inferred ancestral presence/absence patterns) for all sRNAs and their coevolution partners along the Listeria reference tree (see an example in Fig. 4).

\section{The Listeria sRNA-coding gene coevolution hub}

The inferred sRNA-coding genes coevolving network (see Fig. 4b) reveals interesting features. We observed a hub of 12 sRNAs (rli107, rli117, rli123, rli133, rli146, rli26, rli30, rli33-3_rli33, rli34, rli49, rli74 and rli79) that are connected through common coevolution partners. This cluster includes mainly distant (i.e. distance $>40 \mathrm{~kb}$ ) 5'UTRs and CDS coevolving partners, with the exception of partners of only two sRNAs: rli30 paired to CDS partners $l m o 0501$ to lmo0508, and rli74 with its CDS partner $\mathrm{mpl}$ (lmo0203). This cluster includes many genes encoding cell wall proteins, proteins involved in secondary metabolism and virulence factors (see next section and rli133 case study for details). The other 11 sRNAs are all included in 11 individual clusters that contain either 5'UTR coevolving regions (rli132, rli116) exclusively or CDS coevolving regions (rli28-3, rli99_rli140, rli141) exclusively or a mix of both 5'UTRs and CDS regions (rli75, rli5-rliA_rli121, rli34-2, rli115, rli125_rli8-rliC_rli85, rli48). Interestingly, nine out of 11 of these individual clusters include evolving partners that are close on the genome $(<8 \mathrm{~kb})$. Two individual coevolving groups [rli28-3/lmo0035] and [rli5-rliA_rli121/ lmo2309-lmo2407] were found with distant coevolution partners (distance $>800 \mathrm{~kb}$ for both clusters). To summarize, our results reveal an sRNA hub including mainly distantly located coevolving 5'UTRs and CDS regions, some of them exhibiting functions related to Listeria pathogenicity. Most of the remaining coevolution clusters include pairs of sRNA and 5'UTRs/CDS that exhibit very close genomic positions, i.e. a distance between the start of their sRNA and the start of their 5 'UTR/CDS under $8 \mathrm{~kb}$.

We investigated the functional classes of genes coevolving with Listeria regulatory sRNAs by using annotations from the Clusters of Orthologous Groups (COGs) database [42] retrieved from the Listeriomics website [36]. The distribution of coding genes in COG categories reveals a significant functional enrichment of coding genes associated with cell wall or membrane biogenesis (see Table 1, Fisher exact test [43], $p$-value $=0.0131$ ). Interestingly, among coding genes coevolving with Listeria sRNAs, we found seven internalin genes (out of an expected 26 in Listeria [36]), two coding genes of the Listeria Pathogenicity Island LIPI-1 $\mathrm{mpl}$ (lmo0203) and orfX (lmo0206) [44, 45], one component of the flagellar biosynthesis pathway, eight genes involved in secondary metabolism and bacteriophage genes (see Additional file 4: Table S4 for details).

\section{rli133, an sRNA coevolving with genes known to be involved in pathogenicity}

The detailed analysis of rli133 phyletic pattern (Fig. 5a) reveals an early acquisition event in L. monocytogenes common ancestor. Rli133 cannot be found in other Listeria species, indicating that this sRNA is specific to L. monocytogenes species. Nevertheless, rli133 is lost in four strains of lineage III (L. monocytogenes FSLF2-208, $L$. monocytogenes LM850658, L. monocytogenes M7, L. monocytogenes L99) and in the L. monocytogenes FSLJ1208 strain of lineage IV. In these five strains, the corresponding intergenic region is missing due to the insertion of two genes. These genes appear to be specific of these five strains and do not have any homolog in public databases (see Additional file 5: Figure S1). In genomes where rli133 is present, the corresponding sequence is well-conserved and includes few mutation events, i.e., six transitions, two transversions and two indels events corresponding to 12 variables sites out of 126 (9.6\%) in the rli133 alignment (see Additional file 6: 


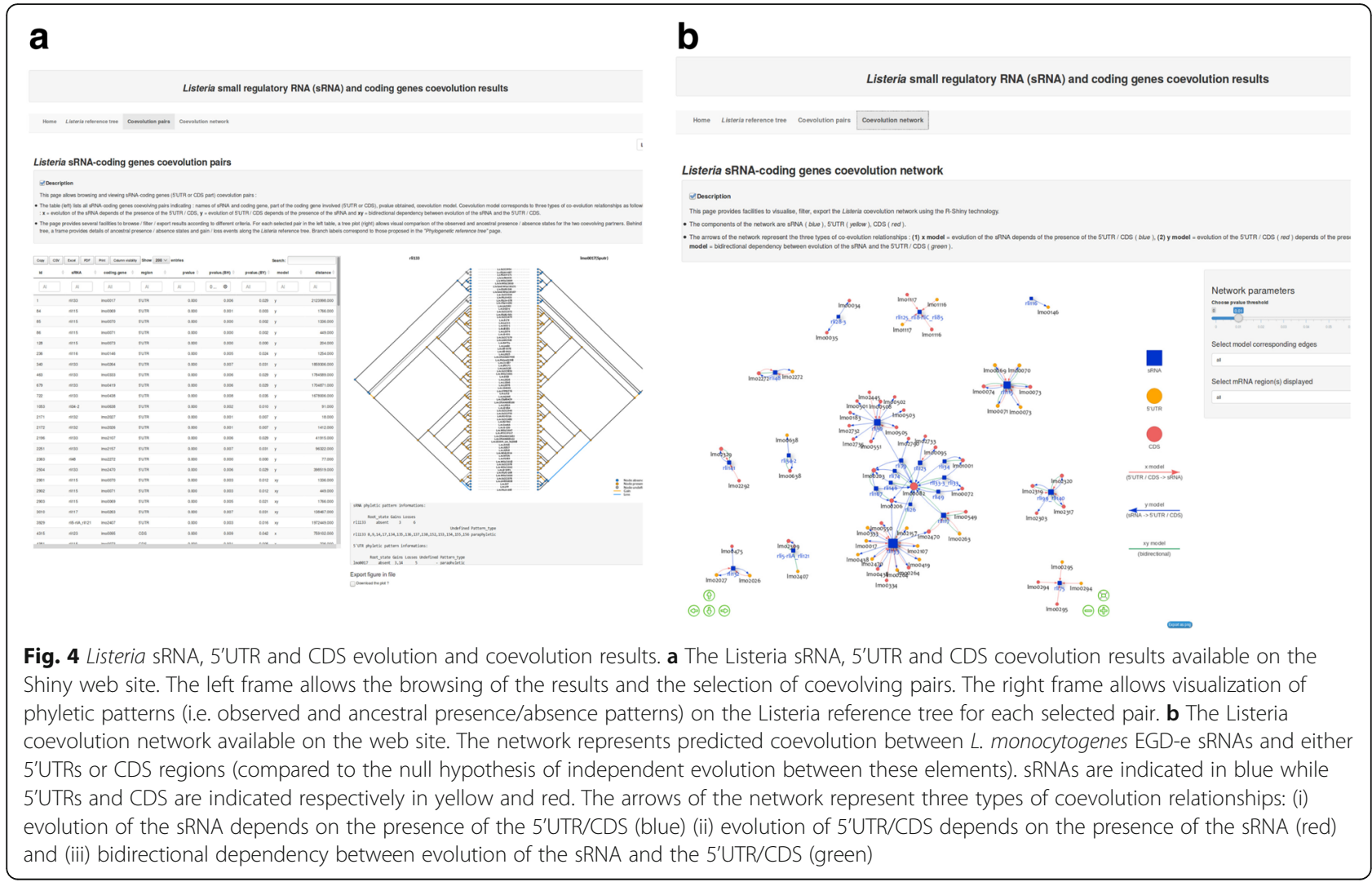

Figure S2). Considering these mutated sites, rli133 homologous sequences can be easily separated in two clusters that correspond to Listeria lineage I and II. Rli133 presents coevolutionary relationships with 12 coding genes, eight $5^{\prime}$ UTRs and seven CDS regions. Coevolving gene partners include three internalin genes: inlI (lmo0333), inlE (lmo0264) and inlP (lmo2470). Internalins are important virulence factors $[46,47]$. InlE may contribute to host tissue colonization $[46,48]$ and $\operatorname{InlP}$ has recently been shown to promote placental infection

Table 1 Functional enrichment of sRNAs and coding genes coevolution groups

\begin{tabular}{|c|c|}
\hline Functional category & $P$ value \\
\hline Amino acid transport and metabolism & 0.9055 \\
\hline Carbohydrate transport and metabolism & 0.2202 \\
\hline Cell wall/membrane biogenesis & $0.0131^{*}$ \\
\hline Energy production and conversion & 0.8563 \\
\hline Replication, recombination and repair & 0.8610 \\
\hline Secondary metabolites biosynthesis, transport and catabolism & 0.5206 \\
\hline Signal transduction mechanisms & 0.3669 \\
\hline Transcription & 0.2648 \\
\hline
\end{tabular}

[47]. The role of inlI in pathogenicity remains to be determined [49]. Interestingly, we found three other sRNAs potentially coevolving with internalin genes: rli117 (lmo0549, lmo0263 and lmo2470), rli30 (lmo2445) and rli132 (lmo2017). Other genes were found to coevolve with rli133, e.g., $\ln t A, \operatorname{lmo0206}$ and $\operatorname{sep} A$. The virulence factor $\ln t A$ (lmo0438) targets the chromatin repressor $B A H D 1$ to activate interferon-stimulated genes in the host cell nucleus [50]. Expression of LntA seems to be tightly controlled to subvert immune responses and prevent antibacterial responses [50]. The orf $X$ (lmo0206) gene is located within the L. monocytogenes pathogenic island 1 (LIPI-1), which includes genes required for Listeria intracellular lifestyle such as $h l y, p l c A$, $p l c B$ and act $A$ [33] and contributes to bacterial survival in macrophages. SepA (lmo2157) encodes a protein involved in septum formation and play a role in stress response [51]. To summarize, six out of the twelve coevolution partners of rli133 exhibit a documented function linked to either pathogenicity, interaction with host cells or stress response [36, 46, 47, 51]. Moreover, rli133 sRNA was found to be expressed in several transcriptomes during infection, especially in blood and intestine $[5,22,36]$. Coevolution between sRNAs and coding genes may be resulting from the existence of direct or indirect functional links. Direct functional links can be explained by physical interaction through base- 


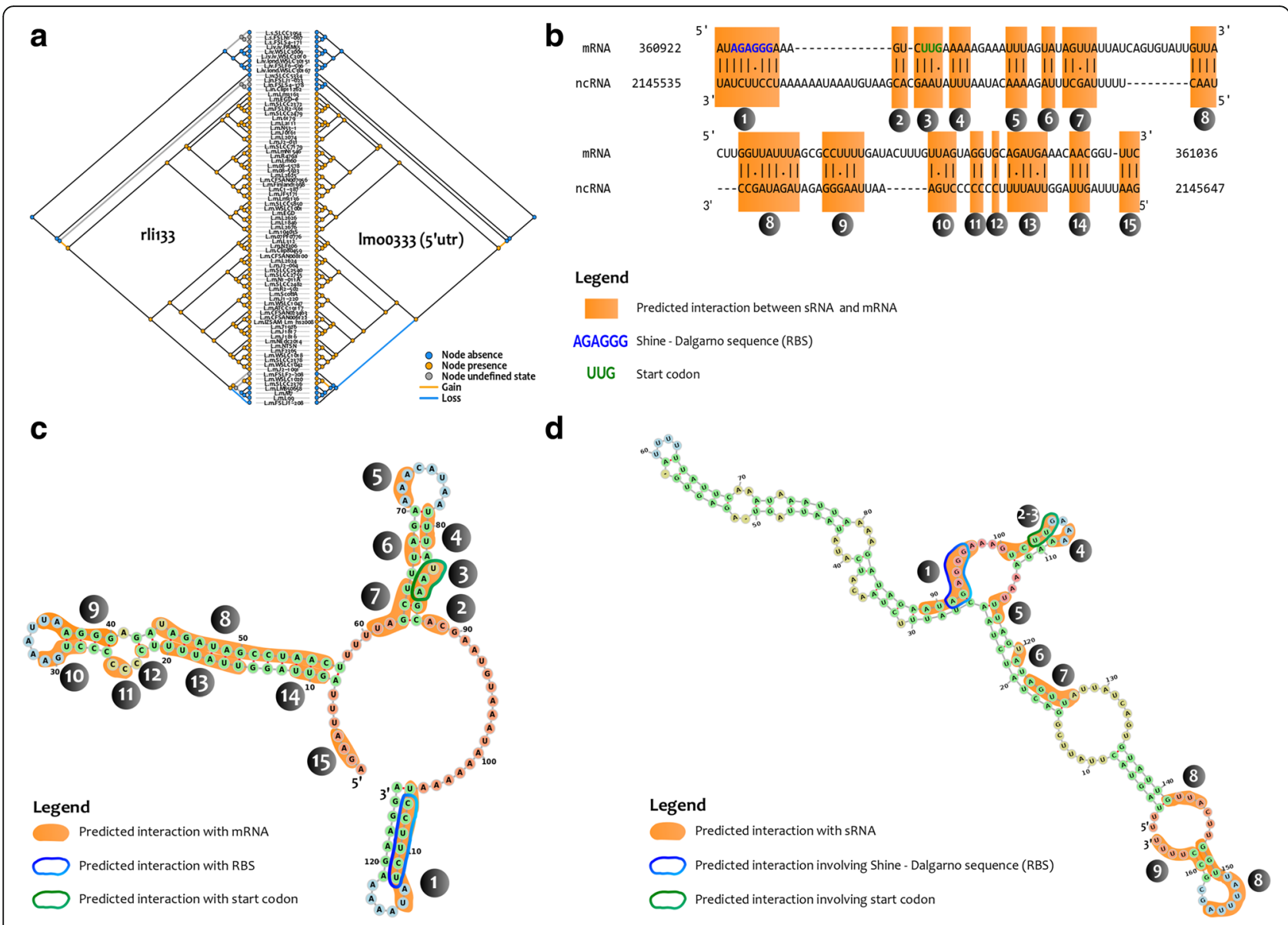

Fig. 5 The rli133 coevolution ties. rli133 shows significant coevolution with six CDS and nine 5'-UTR regions. Figures 5a to 5d show an example of a coevolution pattern and a putative mechanism of interaction between rli133 and the Imo0333 5'UTR region corresponding to the promoter of an Internalin Intl protein. a Coevolution patterns observed between rli133 (left) and the 5'UTR of Imo0333 (right). Yellow circles correspond to observed (or ancestral) sRNA/CDS presence. Blue circles correspond to observed (or ancestral) sRNA/CDS absence. A yellow branch indicates a sRNA/CDS gain event while a blue branch indicates a loss event along the branch. b Predicted interaction regions between rli133 and Imo0333. The figure presents the interaction regions between the $5^{\prime} \mathrm{UTR}$ (and the beginning of the coding region) and the sRNA. Highlighted and numbered regions correspond to predicted interaction zones according to the RNAplex software. c Predicted structure of the sRNA rli133. Structure was generated using LocaRNA software and a multiple alignment of all conserved rli133 sRNAs in the genomic dataset. Highlighted numbered regions correspond to Lmo0333-5'UTR predicted interaction zones of Fig. 5b. Representation of structure was performed with FoRNA. d Predicted structure of the 5'UTR region of Lmo0333. Structure was generated using LocaRNA and a multiple alignment of all conserved Lmo0333 5'UTRs in the genomic dataset. Highlighted numbered regions correspond to rli133 predicted interaction zones of Fig. $5 \mathrm{~b}$. Representation of structure was performed with FoRNA

pairing at specific regions of a sRNA with their target mRNA. To identify possible physical interaction between rli133 and its coevolution partners, we used several methods to predict structure of both the sRNAs and the 5 'UTRs/CDS interacting regions and look for putative interacting zones (see Methods).

We found regions possibly interacting with rli133 for all the 12 genes coevolving with rli133. Nine of these genes were identified to present interacting regions compatible with a negative regulation mechanism: inlI (lmo0333), inlE (lmo0264), inlP (lmo2470), lntA (lmo0438), orfX (lmo0206), lmo0082, lmo0334, lmo0550 and lmo2107. For the three remaining coevolving genes (lmo0419, lmo0017 and lmo2157) we did not identify a consistent interacting region. As illustrated in Fig. 5b, fifteen interacting regions were predicted between lmo0333-5'UTR and rli133. Two interacting regions were overlapping in the proximity of the Shine-Dalgarno sequence and the initiation codon (see regions 1 and 3 in Fig. 5b, c and d), which are crucial sites for ribosome recruitment during the initiation of the translation. On the mRNAs, these sequences are mainly found to be accessible in loops or pseudo-loops (Fig. 5d), suggesting that they are constitutively available for translation. Interaction with complementary regions on sRNAs potentially makes them unavailable for ribosome binding 
during translation initiation, suggesting a potential inhibitory action of rli133 at posttranscriptional level on these genes. The existence of direct interaction between rli133 and coevolving genes partners could explain their coevolution.

To conclude, interacting regions corresponding to putative translation inhibition regions targeted by rli133 were identified for nine coevolving genes including inlE [46, 48], inlI [49], inlP [47], lntA [50] and orf $X$ which were already found to be involved in host-interaction in previous studies.

\section{Discussion}

We built a robust workflow that provided new insights on Listeria sRNA evolution and coevolution patterns. First, the screening of sRNA presence-absence patterns suggests that 60 out of $112 \mathrm{~L}$. monocytogenes EGD-e sRNAs (53\%) shape the Listeria sRNA core set, in the sense that they were found to be present and conserved in all Listeria genomes. These 60 sRNAs were hence inferred present in the common ancestor of Listeria, suggesting that they were present early during the evolutionary history of the genus. This is a lower proportion compared to the 60 out of 83 E. coli K12 sRNAs (72\%) that were found present and conserved in a dataset of 27 complete genomes of E. coli-Shigella [29]. However, this is consistent with the higher number of genomes and the wider evolutionary scale (genus) used in our analysis compared to the species level of the E.coli-Shigella study.

The 52 remaining L. monocytogenes EGD-e sRNA loci constitute the variable sRNA set that is part of the Listeria accessory genome. This number is higher than the 43 accessory Listeria sRNAs previously identified by Kuenne et al. [38] in a smaller dataset of 11 genomes restricted to $L$. monocytogenes species. We found a higher proportion of them exhibiting complex paraphyletic distribution compared to the E. coli/Shigella study: 47 variable sRNAs out of 52 were shown to have paraphyletic pattern (90\%) compared to 25 out of $32(78 \%)$ in the 27 genomes of E. coli-Shigella [29]. Only three and two sRNAs have monophyletic and polyphyletic patterns, respectively. This indicates complex and various evolutionary histories underlying diverse origins and a potentially wide panel of sRNA acquisition and loss mechanisms in Listeria.

Detection of coevolution and analysis of the Listeria sRNA-coding genes coevolving network highlighted many interesting features.

We revealed an evolutionary link between sRNAs and coding genes related to pathogenicity and interaction with the host cell that suggests a key role for these sRNAs to shape Listeria virulence and adaptation. More precisely, we identified a hub of 12 sRNAs (rli26, rli30, rli33-3_rli33 locus, rli34, rli49, rli74, rli79, rli107, rli117, rli123, rli133 and rli146) coevolving with many genes encoding cell wall proteins, especially internalins, that are known to be involved in host cell interaction [52], proteins involved in secondary metabolism, stress response and virulence factors. We detected a significant coevolution pattern of four sRNAs (rli117, rli30, rli132 and rli133) and seven internalin genes (lmo0549, lmo0263, Imo2470, lmo2445, Imo2027, Imo0333 and Imo0264), indicating a probable key functional role of these sRNAs on these genes, possibly regulatory. To our knowledge, the relationship between small regulatory RNAs and internalin evolution was never observed before and opens several new perspectives concerning the possible impact of sRNAs in Listeria evolution and virulence. These results are consistent with previous observations that several internalin genes present long 5 'UTRs that may also be post-transcriptionally regulated and that Listeria controls many of its virulence genes by a mechanism that involves 5'UTRs [23].

Interestingly, previous studies performed in E. coli or $S$. enterica have shown that sRNAs are often found to control the expression of cell wall proteins, particularly in outer membrane [53] or lipopolysaccharide layer synthesis [54]. This is consistent with our result revealing that the 'cell wall or membrane biogenesis' functional category is significantly overrepresented in Listeria sRNAs coevolving genes. Namely, we found seven internalin genes and two coding genes of the Listeria Pathogenicity Island LIPI-1 ( $m p l$, orf $X$ ) in the Listeria sRNA coevolution partners. These results suggest a possible key regulatory role of some Listeria sRNAs on genes involved in host-bacteria interaction and pathogenicity.

We focused on rli133, a L. monocytogenes-specific sRNA, and identified 6 out of 12 rli133 coevolution partners exhibiting a function linked to either pathogenicity, interaction with the host cells or stress response. Interacting regions compatible with mechanisms of mRNA translation inhibition were predicted for rli133 and nine coevolving genes, including inlE [46, 48], inll [49], inlP [47], $\ln t A$ [50] and $\operatorname{orf} X$. These results suggest a possible direct negative regulatory role of rli133, which potentially impairs the translation process of some of its coevolving partners. The presence of compatible interacting regions is not a feature specific to genes coevolving with rli133, but taking together the observations of coevolution pattern and the presence of a consistent interacting zone argue in favor of a functional link. Moreover, we looked for the presence of the nine 5'UTR-interacting zones of the genes co-evolving with rli133 in 5'UTRs and CDS that do not coevolve with rli133 and found only two similar regions for the inlP (lmo2470) interacting zone: one located in another internalin 5'UTR region (inlP/lmo2027) and one located in the 5'UTR region of the lmo0974 gene that is involved in LPS synthesis and 
conserved in all the genomes of the dataset. This argues for quite a good specificity of the rli133 predicted interacting zones. For coevolving partners in which no clear mechanism were highlighted, such as sepA (lmo2157) [51], a well-known stress response gene involved in septum formation, coevolution patterns may correspond to presence of direct interaction at post-translational level or indirect functional links involving intermediate genes. These results suggest that rli133 could act as a negative regulator of genes involved in Listeria pathogenicity.

Interestingly, rli133 sRNA is missing in the L. monocytogenes M7 and L. monocytogenes L99 genomes of $L$. monocytogenes lineage III that also have a reduced internalin-coding genes content (respectively 17 and 18 internalins) [55-57]. This suggests a possible link between the presence of rli133, the internalin gene content and the regulation of pathogenicity. The situation may be more complicated in other genomes such as the pathogenic strain L. monocytogenes J1-208 (lineage IV) identified in goat and whose chromosome contains only 16 internalin-coding genes and no rli133 sRNA. This strain includes a plasmid (pLMIV) which contains additional internalins that may be involved in another mechanism of regulation of pathogenicity [57]. This indicates that the presence of rli133 is not an absolute hallmark for pathogenesis and that other, yet unannotated sRNAs may interact with internalin genes in pathogenic strains of lineage IV. Additional genomes and sRNA experimental datasets are needed in this clade to fully understand the role of sRNAs and internalin coding genes in Listeria pathogenicity.

The Listeria coevolution network also pointed out 11 sRNAs exhibiting correlated evolution, mostly with close 5'UTRs and CDS regions. Screening for distances between coevolving sRNAs and genes indeed revealed two trends concerning gene location: on one hand, genes close to the corresponding sRNA (putative cis-regulated genes closer than $8 \mathrm{~kb}$ ), and on the other hand, genes found at distant locations (putative trans-regulated genes with distances higher than $40 \mathrm{~kb}$ ) (see Additional file 7: Figure S3). One possibility is that some of the closer coevolving sRNAs may correspond to uncharacterized or unannotated 5'UTR regions.

\section{Conclusion}

The analysis of the Listeria coevolving network sheds light on several sRNAs which might play a role in virulence regulation. Since our approach makes it possible to obtain a list of sRNAs present only in the virulent strains, this study paves the way for new biochemical and biological analyses aimed at identifying and deciphering new factors involved in virulence.
The workflow proposed in this work is resourceful and, to our knowledge, does not have any equivalent in previous work. Our strategy proposes several methodological enhancements and additional analyses compared to the pioneer work of Skippington and Ragan [29]. For instance, our strategy was designed to deal with uncovered regions of draft genomes and paralogy problems (both for sRNAs and coding genes). Moreover, three key steps of our workflow, i.e. the reference tree construction, the inference of ancestral presence-absence states and the detection of coevolution between sRNAs and coding genes, rely on the statistical framework of continuous-time Markov models and maximum likelihood, improving on parsimony approaches that do not provide consistent branch length estimation and may lead to lower precision.

Another key advantage of our approach is its extensively generic character since it can be transposed to any type of organism, any type of functional data and, more generally, to any kind of qualitative trait. For example, the strategy developed may be used to look for a possible coevolution between regulatory or structural RNAs and any type of element or feature such as pathogeny islands, pseudogenes, CRISPRs, phages, insertions sequences, etc. The entire workflow is built on an open source frame that is flexible, optimized and implements parallel and distributed computation, while however remaining computationally demanding.

Several features may be proposed in the future to enhance the proposed strategy. First, as we currently only consider presence, absence and unknown states, it constitutes an oversimplification of the way functional elements are defined, also undermining paralogy for sRNA or coding genes. Consequently, an enhancement of our strategy would be to deal with the occurrence of sRNAs and coding genes for both evolution and coevolution analysis. Second, another useful extension of the current strategy would be to include the analysis of mutation patterns and coevolving sites also for the core sRNAs and coding genes present in all the genomes of the dataset as well. This could be performed by including an additional step in the workflow that relies on a previously published method like the CoMap software [58].

\section{Methods}

\section{Listeria genome dataset}

Seventy-nine complete public genomes of Listeria were obtained from GenBank (release 211). A full description of the 79 Listeria genomes is available in Additional file 1: Table S1). The dataset includes 70 complete and $9 \mathrm{draft}$ genomes representing five different Listeria species $(L$. monocytogenes, $L$. ivanovii, $L$. inoccua, $L$. welshimeri and $L$. seeligeri). L. monocytogenes genomes were the most represented (73 genomes corresponding to $92 \%$ of our dataset). 


\section{Listeria monocytogenes EGD-e sRNA}

A set of 304 experimentally validated sRNAs from $L$. monocytogenes EGD-e was extracted from the Listeriomics database [36]. We focused on the 154 sRNAs annotated as putative trans sRNAs which are known as important regulators of gene expression in bacteria acting on independently expressed targets. A group of 19 sRNAs were excluded because they recently have been found to include small ORFs [6]. Overlapping sRNAs and sRNAs harboring paralogs in the L. monocytogenes EGD-e genome were processed using the following procedure. sRNAs were aligned on the L. monocytogenes EGD-e genome sequence using BLASTN+ [59]. Overlapping hits were merged considering a minimal overlap length of $15 \mathrm{pb}$, independently of their orientation. Finally, 112 sRNA loci were used as input sequences in the following analyses, including 15 loci built from merged hits and 97 original sequences.

\section{sRNA and coding gene coevolution strategy}

The strategy we developed is implemented in a Snakemake workflow [60] that consists in four main steps (see Fig. 1).

\section{Step 1: Phylogenetic reference tree.}

PanOCT, version 3.23 [61], was used to build groups of orthologs from annotated genomes. PanOCT is able to deal with recently diverging paralogs by using neighborhood gene information (synteny). All the parameters were set to default values except for the length ratio to discard shorter protein fragments when a protein is split due to a frameshift or other mechanisms was set to 1.33 as recommended by the authors. Amino-acid sequences of ortholog families were then aligned using ProbCons, version 1.12 [62], and resulting alignments were postprocessed using GBLOCKS, version 0.91b [63], using the following parameters: the minimum number of sequences for a conserved position was set to $(n / 2)+1$, the minimum number of sequences for a flank position to $(n / 2)+1$ (where $n$ is the total number of sequences in the aligned dataset), the maximum number of contiguous non-conserved positions was set to 20, the minimum length for a block to 5 , and gap positions were allowed [8].

The reference tree was built using the syntenic core gene families corresponding to the PanOCT clusters with a single unique ortholog in each genome of our dataset. The corresponding nucleic acid alignments were obtained from all these core families filtered amino-acid alignments and concatenated into a single superalignment to compute a maximum likelihood tree using FastTree2, version 2.1.9 [64]. The following parameters were used for FastTree2: the Generalized Time-Reversible model (GTR) was chosen, the likelihood was reported under the Gamma model using 20 categories of sites, the exhaustive search mode ("-slow" option) was selected to obtain a more accurate reconstruction, NNI and SPR heuristics were used to browse the tree space. Support analyses were performed using Shimodaira Hasegawa test (SH) and 1000 resampling steps of site likelihood.

Step 2: sRNA and coding gene presence-absence matrix.

Presence-absence patterns were inferred from BLAST analyses with different parameters for sRNAs and coding genes. L. monocytogenes EGD-e sRNAs were aligned on the genome dataset using BLASTN+, version 2.2.29 [59]. Resulting hits were filtered using two criteria: an e-value $<10^{-2}$ and a coverage related to the query sequence $\geq 70 \%$. Only sRNAs meeting these two criteria were considered as present in the targeted genomes.

For coding genes, we analyzed separately 5'UTRs and CDS regions. L. monocytogenes EGD-e CDS were retrieved from GenBank annotations and aligned against all Listeria genomes using BLASTP+, version 2.2.29 [59]. Resulting hits were filtered using the following criteria: an e-value $<10^{-2}$, a coverage relative to the query sequence $\geq 70 \%$, an identity rate $\geq 60 \%$ and a bitscore $\geq 50$. Only CDS meeting these three criteria were considered as present in the targeted genomes.

L. monocytogenes EGD-e 5'UTRs were retrieved using the following procedure. When available, we used experimental data indicating 5'UTR positions [36] to extract the corresponding DNA sequence. When not available, 5'UTR positions were defined arbitrarily as the 100 nearest 5 ' nucleotides upstream from each $L$. monocytogenes EGD-e CDS start codon of intergenic region. Only 5'UTRs with a minimum size of 15 bp were kept. 5'UTR sequences were then used as queries for BLASTN+ [59] alignments against all genomes. Resulting BLASTN+ hits were filtered using the following criteria: an e-value $<10^{-2}$, and a minimal identity percentage and coverage adjusted to the 5'UTR sequence lengths as follows. For 5'UTRs with lengths from 15 to $20 \mathrm{bp}$, the minimum identity percentage was set to $90 \%$ and the minimum coverage percentage to $100 \%$. For $20-50$ bp long 5'UTRs, both identity percentage and coverage were set to a minimum of $80 \%$. For $50-100$ bp and $>100$ bp long 5 'UTRs, the minimal identity percentage was set to $80 \%$ and the minimal coverage was set to $50 \%$ and $25 \%$, respectively. 5'UTRs meeting these criteria in subject genomes were considered as present.

A binary vector $(0 / 1)$ corresponding to the absence/ presence profile in the whole genome dataset was finally generated using BLASTN+ results and filters defined above. Due to their lack of informative value, sRNAs, 5'UTRs and CDS found in all genomes were not taken into account in subsequent analyses. 
To avoid absence mispredictions corresponding to unsequenced regions of draft genomes, absence events of non-coding elements (sRNAs and 5'UTRs) were systematically checked as follows: for queries without hit in a given genome, $5^{\prime}$ and $3^{\prime}$ adjacent genes of the query element were screened for putative homologs in the same genome. In case where homologs were found, the non-coding sequence between the two homolog genes was extracted and screened for stretches of Ns, i.e. assembly gaps. If such stretches were found, the state of the query element was considered as undetermined due to missing DNA region in the considered genome ('?' state assigned). If the region was present but not similar to the query sequence, the query element was consider to be absent ('0' state assigned).

Step 3: Ancestral presence-absence pattern reconstruction.

Presence/absence ancestral states were reconstructed using the recent "Hidden rates model" method proposed by Beaulieu et al. [65]. This method uses Hidden Markov Models (HMM) to reconstruct ancestral character states from observed states and a reference phylogenetic tree. It makes it possible to use different transition rate classes. We used the 'rayDISC' function of the 'corHMM' $\mathrm{R}$ package version 1.20 [17] and selected the 'ARD' transition model, i.e. independent transition rates. Internal node states were inferred using maximum likelihood estimation and joint probabilities. The root state probabilities were inferred using the method of Fitzjohn and Maddison [66]. Initial transition rates were estimated using the results of PanOCT orthologs obtained in Step 1 and computed using the 'DiscML' function of the 'DiscML' R package, version 1.0.1 [67], and the 'ARD' transition model (assuming independent transition events, in this case, gain and loss events, for each element). This step results in a matrix containing the binary presence/absence $(0 / 1)$ pattern for each internal node of the reference tree and for the three analysed features (sRNAs, CDS, 5'UTRs). Finally, gain and loss events were determined as follows: if the feature was absent (present) in a given node but present (absent) in its ancestor, it was considered as lost (gained) along the corresponding branch linking both nodes.

Step 4: Detection of coevolution events.

Our strategy allows the detection of coevolution between a sRNA and a 5'UTR or CDS using a reference phylogenetic tree and both observed and ancestral presence-absence patterns. We used the 'corDISC' function of the 'corHMM' $\mathrm{R}$ package [65] to identify putative coevolutionary relationships. This function fits Pagel's models of independency and dependency [39] to identify dependent evolution between two binary characters (in this case, the presence or absence of sRNAs, CDS/
5'UTRs) and related to a phylogenetic tree. The first model supports an independent relationship between both binary traits: sRNA and 5'UTR/CDS (the null hypothesis). The second kind of model (the alternative hypotheses) supports a dependent relationship between both traits (coevolution). The use of ancestral states along the phylogenetic reference tree makes it possible to evaluate the probable temporal ordering of changes between two $\mathrm{x}$ and $\mathrm{y}$ presence/absence patterns and to test hypotheses about cause and effect. For this, we used three kinds of dependency models: the $x$ model, meaning that the evolution of the sRNA depends on the presence/absence state of the 5'UTR/CDS, the $y$ model, meaning that the evolution of 5'UTR/CDS depends on the presence/absence state of the sRNA and the $x y$ model, assuming bidirectional dependency between evolution of the sRNA and the 5'UTR/CDS element.

The 'corDISC' function merges the two $\mathrm{x}, \mathrm{y}$ traits in a single vector, fits them on a precomputed phylogenetic tree using a specified model and then returns the likelihood of the model. The likelihood of each model was computed and a Likelihood Ratio Test (LRT) was performed. The corresponding $p$-value was computed. All of the analyses were performed between each variable sRNA, each variable CDS and 5' UTR. P-values were corrected for multiple testing using the BenjaminiHochberg (BH) procedure [68]. Finally, we only retained coevolving pairs of sRNA loci and coding gene elements (5'UTR and CDS) with a minimum $\mathrm{BH}$ corrected $p$-value threshold of 0.01 .

The coevolution network between sRNAs and coding genes was reconstructed using inferred significant dependency relationships between phyletic patterns of sRNAs and coding gene elements (5'UTR and CDS) of L. monocytogenes EGD-e. Graph representations were built using the 'igraph', version 1.0.1 [69], 'visNetwork', version 1.0.3 [70], and 'Shiny', version 1.0.1 [41] R packages.

\section{Gene targets functional enrichment}

Functional enrichment test was performed using $L$. monocytogenes EGD-e gene COG ontologies [36] and computed using a Fisher's exact test [43] ('fisher.test' function from the 'stats' $\mathrm{R}$ package, version 3.5.0 [71]), with a $p$-value threshold of 0.05 .

\section{Interacting regions prediction}

Possible physical interactions between sRNAs and coding genes identified as coevolving partners using our method were predicted using several pieces of software: Ssearch (implementation from Wisconsin Package), version 6.1, IntaRNA, version 2.0.2, RIsearch, version 1.1, RNAcofold, version 2.3.4 and RNAplex, version 2.3.4 
[72-76], which are all included in the sRNAtabac resource [77]. We used an extended region including $100 \mathrm{bp}$ before and after the start codon to identify putative interactions between a sRNA region and the extended 5'UTR region of mRNAs (original regions were used for CDS). Only interactions containing a minimum of six successive interacting matches were selected and considered as valid.

Homologous sequences of rli133 previously identified (see Step 2 for details) in L. monocytogenes genomes were extracted. Homologous sequences of $1 \mathrm{mo0} 0333$ (inlI) 5'UTR (see Step 2 for details) were extended up to 60 nucleotides after the start codon. Rli133 and lmo0333extended 5'UTR sequences were processed using LocARNA software, version 1.8.9 [73]. LocARNA is a tool that allows simultaneous folding and alignment of input RNA sequences. LocARNA default alignment accuracy was increased using match probabilities and probabilistic consistency transformation. Additional parameters were used since it is recommended by the authors in the software documentation for aligning up to about 15 sequences of lengths up to a few hundred nucleotides. The weight of base pair match contribution was set to 400. An iterative refinement of the progressive alignment was performed using two iterations. The 2D structure representation of rli133 and lmo0333- extended 5'UTR were computed with FoRNA, version 0.1 [78], using consensus structures of rli133 and the lmo0333-extended 5'UTR associated with corresponding sequences of the reference strain $L$. monocytogenes EGD-e.

\section{Additional files}

Additional file 1: Table S1. List of the 79 genomes used in this study. The table includes the list of 79 genomes obtained from Listeriomics and retrieved from the NCBI database. Several fields have been abbreviated for easier reading: 'Se.': strain serotype, 'Li.': Listeria lineage and 'Co.': country where the strain was first isolated. (DOCX $98 \mathrm{~kb}$ )

Additional file 2: Table S2. List of the 112 sRNA loci used in this study.The table includes 97 sRNAs obtained from the Listeriomics database and 15 merged regulatory sRNA loci tagged with an * in the table and obtained from the procedure described in Methods. (XLSX $21 \mathrm{~kb}$ )

Additional file 3: Table S3. Ancestral presence/absence patterns of L.m. EGD-e regulatory sRNAs. For all 52 L.m. EGD-e variable sRNAs, the table includes the following information according to the Listeria reference tree (see Fig. 2): root presence - absence information (Root state column), tree branches labels where gain (Gains column) and loss events (Losses column) were inferred (labels correspond to branch identifiers indicated in the cladogram of Fig. 2). The undefined column corresponds to tree branch labels with undefined state due to missing data in the corresponding genomes (draft genomes). The pattern_type column corresponds to the three different types of phyletic profiles inferred: monophyletic, polyphyletic or paraphyletic profiles. (DOCX $128 \mathrm{~kb}$ )

Additional file 4: Table S4. Listeria sRNAs and coding genes coevolution groups. For each sRNA, the table includes the following informations on the corresponding co-evolving elements: the gene locus tag name ('Element' column), the type of element (CDS or 5'UTR, 'Type' column), the type of dependency model that highlighted the interaction: $x=$ evolution of the sRNA depends on the state of the $5^{\prime} U T R / C D S$, $y=$ evolution of $5^{\prime} U T R / C D S$ depends on the state of the sRNA and $x y=$ bidirectional dependency between evolution of the sRNA and the 5'UTR/CDS element (Model column), the distance between the sRNA and the element in nucleotides (Distance column) and the description of the gene/operon function according to Listeriomics database ('Description' column); 'id' = identical content. Coevolution groups that are included in the main network hub are highlighted in gray. (DOCX $142 \mathrm{~kb}$ )

Additional file 5: Figure S1. Rli133 genomic context conservation in Listeria. 5' $^{\prime}$ and $3^{\prime}$ homolog genes are represented using red arrows. GFXXXX names correspond to PanOCT ortholog clusters identifiers. Blue arrows correspond to two genes inserted in several strains of Listeria lineages III and IV. (PDF $212 \mathrm{~kb}$ )

Additional file 6: Figure S2. Multiple alignment of rli133. This figure represents the multiple alignment of rli133 sequences in strains where it is present. Red denotes a fully conserved position. The phylogenetic tree at the left corresponds to a Maximum Likelihood tree computed from the corresponding multiple alignment. (PDF $1413 \mathrm{~kb}$ )

Additional file 7: Figure S3. Genomic distance between coevolving sRNAs and CDS. Plain curves show the distance density between sRNAs and $5^{\prime}$ UTRs (red) or CDS (blue) engaged in coevolution relationships, considering genome circularity. They are compared to distances between sRNAs and all 5'UTRs or CDS (all) respectively represented by red and blue dotted curves. (PDF $117 \mathrm{~kb}$ )

\section{Abbreviations}

5' UTR: 5' Untranslated Region of coding genes; CDS: Coding DNA Sequence; COG: Clusters of Orthologous Group; mRNA: messenger RNA; SH: Shimodaira Hasegawa; sRNA: Small regulatory RNA

\section{Acknowledgments}

We are grateful to the Genotoul bioinformatics platform, Toulouse Midi-Pyrénées, for providing assistance, computing and storage resources.

\section{Funding}

This work received financial support from the French National Research Agency (BacNet Investissement d'Avenir project, 10-BINF-02-01). The funders had no role in study design, data collection and analysis, decision to publish, or preparation of the manuscript.

Availability of data and materials

All data used in this study are publically available (see Additional file 1: Table S1 and Additional file 2: Table S2 for details).

\section{Authors' contributions}

HC and CG designed the study. FC, LM, CH, CG and HC conceived the workflow and FC implemented it. FC, AP, MD, CG and $\mathrm{HC}$ performed the data analysis and FC, LM, CH, AP, AM, MD, OD, CG, PC and HC discussed and interpreted the results. $P C$ coordinated the BacNet project. FC, LM, MD, OD, $\mathrm{CB}, \mathrm{CG}$, and $\mathrm{HC}$ wrote the manuscript. All authors read and approved the final manuscript.

Ethics approval and consent to participate

No permission was required from the ethics committee as the project did not involve testing of human, animal or endangered plant species subjects.

Consent for publication

Not applicable.

Competing interests

The authors declare that they have no competing interests.

\section{Publisher's Note}

Springer Nature remains neutral with regard to jurisdictional claims in published maps and institutional affiliations. 


\section{Author details}

1Université de Toulouse, INRA, UR 875 Unité Mathématiques et Informatique Appliquées de Toulouse, Auzeville, 31326 Castanet-Tolosan, France. ${ }^{2}$ Département de Biologie Cellulaire et Infection, Institut Pasteur, Unité des Interactions Bactéries-Cellules, F-75015 Paris, France. ${ }^{3}$ INSERM, U604,F-75015 Paris, France. ${ }^{4}$ INRA, USC2020, F-75015 Paris, France. ${ }^{5}$ Institut Pasteur Bioinformatics and Biostatistics Hub - C3BI, USR 3756 IP CNRS, Paris, France. ${ }^{6}$ Université Paris Diderot, Sorbonne Paris Cité, F-75013 Paris, France. ${ }^{7}$ Present address: Public Health England, 61 Colindale Avenue, London NW9 5EQ, England.

\section{Received: 26 June 2017 Accepted: 29 October 2017}

\section{Published online: 16 November 2017}

\section{References}

1. Gottesman S, Storz G. Bacterial small RNA regulators: versatile roles and rapidly evolving variations. Cold Spring Harb Perspect Biol. 2011;3 doi:10.1101/cshperspect.a003798.

2. Modi SR, Camacho DM, Kohanski MA, Walker GC, Collins JJ. Functional characterization of bacterial sRNAs using a network biology approach. Proc Natl Acad Sci U S A. 2011;108:15522-7.

3. Mandin P, Guillier M. Expanding control in bacteria: interplay between small RNAs and transcriptional regulators to control gene expression. Curr Opin Microbiol. 2013;16:125-32.

4. Caldelari I, Chao Y, Romby P, Vogel J. RNA-mediated regulation in pathogenic bacteria. Cold Spring Harb Perspect Med. 2013;3:a010298.

5. Toledo-Arana A, Dussurget O, Nikitas G, Sesto N, Guet-Revillet H, Balestrino $D$, et al. The listeria transcriptional landscape from saprophytism to virulence. Nature. 2009;459:950-6.

6. Mellin JR, Cossart P. The non-coding RNA world of the bacterial pathogen listeria monocytogenes. RNA Biol. 2012;9:372-8.

7. Peer A, Margalit H. Accessibility and evolutionary conservation mark bacterial small-rna target-binding regions. J Bacteriol. 2011;193:1690-701.

8. Richter AS, Backofen R. Accessibility and conservation: general features of bacterial small RNA-mRNA interactions? RNA Biol. 2012;9:954-65.

9. Beisel $\mathrm{CL}$, Updegrove TB, Janson BJ, Storz G. Multiple factors dictate target selection by Hfq-binding small RNAs. EMBO J. 2012;31:1961-74.

10. Wright PR, Richter AS, Papenfort K, Mann M, Vogel J, Hess WR, et al. Comparative genomics boosts target prediction for bacterial small RNAs. Proc Natl Acad Sci U S A. 2013;110:E3487-96.

11. Updegrove TB, Shabalina SA, Storz G. How do base-pairing small RNAs evolve? FEMS Microbiol Rev. 2015;39:379-91.

12. Raghavan R, Groisman EA, Ochman H. Genome-wide detection of novel regulatory RNAs in E. Coli. Genome Res. 2011;21:1487-97.

13. Kröger C, Dillon SC, Cameron ADS, Papenfort K, Sivasankaran SK, Hokamp K, et al. The transcriptional landscape and small RNAs of salmonella enterica serovar Typhimurium. Proc Natl Acad Sci U S A. 2012;109:E1277-86.

14. Irnov I, Sharma CM, Vogel J, Winkler WC. Identification of regulatory RNAs in Bacillus Subtilis. Nucleic Acids Res. 2010;38:6637-51.

15. Mars RAT, Nicolas P, Ciccolini M, Reilman E, Reder A, Schaffer M, et al. Small regulatory RNA-induced growth rate heterogeneity of Bacillus Subtilis. PLoS Genet. 2015;11:e1005046.

16. Mellin JR, Koutero M, Dar D, Nahori M-A, Sorek R, Cossart P. Riboswitches. Sequestration of a two-component response regulator by a riboswitchregulated noncoding RNA. Science. 2014;345:940-3.

17. Mellin JR, Tiensuu T, Bécavin C, Gouin E, Johansson J, Cossart PA. Riboswitch-regulated antisense RNA in listeria monocytogenes. Proc Natl Acad Sci U S A. 2013;110:13132-7.

18. Christiansen JK, Nielsen JS, Ebersbach T, Valentin-Hansen P, SøgaardAndersen L, Kallipolitis BH. Identification of small Hfq-binding RNAs in listeria monocytogenes. RNA. 2006;12:1383-96.

19. Mandin P, Repoila F, Vergassola M, Geissmann T, Cossart P. Identification of new noncoding RNAs in listeria monocytogenes and prediction of mRNA targets. Nucleic Acids Res. 2007;35:962-74.

20. Oliver HF, Orsi RH, Ponnala L, Keich U, Wang W, Sun Q, et al. Deep RNA sequencing of $L$. monocytogenes reveals overlapping and extensive stationary phase and sigma B-dependent transcriptomes, including multiple highly transcribed noncoding RNAs. BMC Genomics. 2009;10:641.

21. Mraheil MA, Billion A, Mohamed W, Mukherjee K, Kuenne C, Pischimarov J, et al. The intracellular sRNA transcriptome of listeria monocytogenes during growth in macrophages. Nucleic Acids Res. 2011;39:4235-48.
22. Wurtzel O, Sesto N, Mellin JR, Karunker I, Edelheit S, Bécavin C, et al. Comparative transcriptomics of pathogenic and non-pathogenic listeria species. Mol Syst Biol. 2012;8:583.

23. Loh E, Dussurget O, Gripenland J, Vaitkevicius K, Tiensuu T, Mandin P, et al. A trans-acting riboswitch controls expression of the virulence regulator PrfA in listeria monocytogenes. Cell. 2009;139:770-9.

24. Johansson J, Mandin P, Renzoni A, Chiaruttini C, Springer M, Cossart P, An RNA. Thermosensor controls expression of virulence genes in listeria monocytogenes. Cell. 2002;110:551-61.

25. Sesto N, Koutero M, Cossart P. Bacterial and cellular RNAs at work during listeria infection. Future Microbiol. 2014;9:1025-37.

26. Quereda JJ, Ortega AD, Pucciarelli MG, García-Del Portillo F. The listeria smal RNA Rli27 regulates a Cell Wall protein inside eukaryotic cells by targeting a long 5'-UTR variant. PLoS Genet. 2014;10:e1004765.

27. Peng Y-L, Meng Q- L, Qiao J, Xie K, Chen C, Liu T-L, et al. The regulatory roles of ncRNA Rli60 in adaptability of listeria monocytogenes to environmental stress and biofilm formation. Curr Microbiol. 2016;73:77-83.

28. Toffano-Nioche C, Nguyen AN, Kuchly C, Ott A, Gautheret D, Bouloc P, et al. Transcriptomic profiling of the oyster pathogen Vibrio Splendidus opens a window on the evolutionary dynamics of the small RNA repertoire in the vibrio genus. RNA. 2012;18:2201-19.

29. Skippington E, Ragan MA. Evolutionary dynamics of small RNAs in 27 Escherichia Coli and Shigella genomes. Genome Biol Evol. 2012;4:330-45.

30. Peer A, Margalit H. Evolutionary patterns of Escherichia Coli small RNAs and their regulatory interactions. RNA. 2014;20:994-1003.

31. Orsi RH, Wiedmann M. Characteristics and distribution of listeria spp., including listeria species newly described since 2009. Appl Microbiol Biotechnol. 2016;100:5273-87.

32. Hamon M, Bierne $H$, Cossart P. Listeria monocytogenes: a multifaceted model. Nat Rev Microbiol. 2006;4:423-34.

33. Pizarro-Cerdá J, Cossart P. Subversion of cellular functions by listeria monocytogenes. J Pathol. 2006;208:215-23.

34. Cossart P. Illuminating the landscape of host-pathogen interactions with the bacterium listeria monocytogenes. Proc Natl Acad Sci U S A. 2011;108:19484-91.

35. Glaser P, Frangeul L, Buchrieser C, Rusniok C, Amend A, Baquero F, et al. Comparative genomics of listeria species. Science. 2001;294:849-52.

36. Bécavin C, Koutero M, Tchitchek N, Cerutti F, Lechat P, Maillet N, et al. Listeriomics: an interactive web platform for systems biology of listeria. mSystems. 2017;2 doi:10.1128/mSystems.00186-16.

37. Bécavin C, Bouchier C, Lechat P, Archambaud C, Creno S, Gouin E, et al. Comparison of widely used listeria monocytogenes strains EGD, 10403S, and EGD-e highlights genomic variations underlying differences in pathogenicity. MBio. 2014;5:e00969-14.

38. Kuenne C, Billion A, Mraheil MA, Strittmatter A, Daniel R, Goesmann A, et al. Reassessment of the listeria monocytogenes pan-genome reveals dynamic integration hotspots and mobile genetic elements as major components of the accessory genome. BMC Genomics. 2013;14:47.

39. Pagel M. Detecting correlated evolution on phylogenies: a general method for the comparative analysis of discrete characters. Proc R Soc B Biol Sci. 1994;255:37-45.

40. Orsi RH, den Bakker HC, Wiedmann M. Listeria monocytogenes lineages: genomics, evolution, ecology, and phenotypic characteristics. Int J Med Microbiol. 2011;301:79-96.

41. Beeley C. Web application with R using shiny. Packt Pub Limited; 2013.

42. Tatusov RL. The COG database: a tool for genome-scale analysis of protein functions and evolution. Nucleic Acids Res. 2000;28:33-6.

43. Fisher RA. The logic of inductive inference. J R Stat Soc. 1935;98:39.

44. Vázquez-Boland JA, Domínguez-Bernal G, González-Zorn B, Kreft J, Goebel W. Pathogenicity islands and virulence evolution in listeria. Microbes Infect. 2001;3:571-84.

45. Chatterjee SS, Hossain H, Otten S, Kuenne C, Kuchmina K, Machata S, et al. Intracellular gene expression profile of listeria monocytogenes. Infect Immun. 2006;74:1323-38.

46. Cabanes D, Dehoux P, Dussurget O, Frangeul L, Cossart P. Surface proteins and the pathogenic potential of listeria monocytogenes. Trends Microbiol. 2002;10:238-45.

47. Faralla C, Rizzuto GA, Lowe DE, Kim B, Cooke C, Shiow LR, et al. InIP, a new virulence factor with strong placental tropism. Infect Immun. 2016;84:3584-96.

48. Raffelsbauer D, Bubert A, Engelbrecht F, Scheinpflug J, Simm A, Hess J, et al. The gene cluster inIC2DE of listeria monocytogenes contains additional 
new internalin genes and is important for virulence in mice. Mol Gen Genet. 1998;260:144-58.

49. Sabet C, Lecuit M, Cabanes D, Cossart P, Bierne HLPXTG. Protein InIJ, a newly identified internalin involved in listeria monocytogenes virulence. Infect Immun. 2005;73:6912-22.

50. Lebreton A, Lakisic G, Job V, Fritsch L, Tham TN, Camejo A, et al. A bacterial protein targets the BAHD1 chromatin complex to stimulate type III interferon response. Science. 2011;331:1319-21.

51. Hain T, Hossain H, Chatterjee SS, Machata S, Volk U, Wagner S, et al. Temporal transcriptomic analysis of the listeria monocytogenes EGD-e $\sigma B$ regulon. BMC Microbiol. 2008;8:20.

52. Bierne $\mathrm{H}$, Sabet $\mathrm{C}$, Personnic $\mathrm{N}$, Cossart $\mathrm{P}$. Internalins: a complex family of leucine-rich repeat-containing proteins in listeria monocytogenes. Microbes Infect. 2007;9:1156-66.

53. Vogel J, Papenfort K. Small non-coding RNAs and the bacterial outer membrane. Curr Opin Microbiol. 2006;9:605-11.

54. Klein G, Raina S. Regulated control of the assembly and diversity of LPS by noncoding sRNAs. Biomed Res Int. 2015;2015:153561.

55. Deng X, Phillippy AM, Li Z, Salzberg SL, Zhang W. Probing the pan-genome of listeria monocytogenes: new insights into intraspecific niche expansion and genomic diversification. BMC Genomics. 2010;11:500.

56. Roberts A, Nightingale K, Jeffers G, Fortes E, Kongo JM, Wiedmann M. Genetic and phenotypic characterization of listeria monocytogenes lineage III. Microbiology. 2006;152:685-93.

57. den Bakker HC, Bowen BM, Rodriguez-Rivera LD, Wiedmann MFSL. J1-208, a virulent uncommon phylogenetic lineage IV listeria monocytogenes strain with a small chromosome size and a putative virulence plasmid carrying internalin-like genes. Appl Environ Microbiol. 2012;78:1876-89.

58. Dutheil J, Galtier N. Detecting groups of coevolving positions in a molecule: a clustering approach. BMC Evol Biol. 2007;7:242

59. Camacho C, Coulouris G, Avagyan V, Ma N, Papadopoulos J, Bealer K, et al. BLAST+: architecture and applications. BMC Bioinformatics. 2009:10:421.

60. Köster J, Rahmann S. Snakemake-a scalable bioinformatics workflow engine. Bioinformatics. 2012;28:2520-2.

61. Fouts DE, Brinkac L, Beck E, Inman J, Sutton G. PanOCT: automated clustering of orthologs using conserved gene neighborhood for pangenomic analysis of bacterial strains and closely related species. Nucleic Acids Res. 2012:40:e172.

62. Do CB, Mahabhashyam MSP, Brudno M, Batzoglou S. ProbCons: probabilistic consistency-based multiple sequence alignment. Genome Res. 2005;15:330-40.

63. Castresana J. Selection of conserved blocks from multiple alignments for their use in phylogenetic analysis. Mol Biol Evol. 2000;17:540-52.

64. Price MN, Dehal PS, Arkin AP. FastTree 2-approximately maximumlikelihood trees for large alignments. PLoS One. 2010;5:e9490.

65. Beaulieu JM, O'Meara BC, Donoghue MJ. Identifying hidden rate changes in the evolution of a binary morphological character: the evolution of plant habit in campanulid angiosperms. Syst Biol. 2013;62:725-37.

66. FitzJohn RG, Maddison WP, Otto SP. Estimating trait-dependent speciation and extinction rates from incompletely resolved phylogenies. Syst Biol. 2009;58:595-611.

67. Kim T, Hao W. DiscML: an R package for estimating evolutionary rates of discrete characters using maximum likelihood. BMC Bioinformatics. 2014;15:320

68. Yekutieli D, Benjamini Y. under dependency. Ann Stat. 2001;29:1165-88.

69. igraph - Network analysis software [Internet]. [cited 7 Feb 2017]. Available: http://igraph.org/.

70. datastorm-open. datastorm-open/visNetwork. In: GitHub [Internet]. [cited 9 Feb 2017]. Available: https://github.com/datastorm-open/visNetwork.

71. R: The R Stats Package [Internet]. [cited 11 Apr 2017]. Available: https://stat. ethz.ch/R-manual/R-devel/library/stats/html/stats-package.html.

72. Wenzel A, Akbasli E, Gorodkin J. Rlsearch: fast RNA-RNA interaction search using a simplified nearest-neighbor energy model. Bioinformatics. 2012;28: 2738-46.

73. Gruber AR, Lorenz R, Bernhart SH, Neuböck R, Hofacker IL. The Vienna RNA websuite. Nucleic Acids Res. 2008;36:W70-4.

74. Ropelewski AJ, Nicholas HB, Deerfield DW. Mathematically complete nucleotide and protein sequence searching using Ssearch. Curr Protoc Bioinformatics. 2004;

75. Wright PR, Georg J, Mann M, Sorescu DA, Richter AS, Lott S, et al. CopraRNA and IntaRNA: predicting small RNA targets, networks and interaction domains. Nucleic Acids Res. 2014;42:W119-23.
76. Womble DDGCG. The Wisconsin package of sequence analysis programs. Methods Mol Biol. 2000;132:3-22

77. Toulouse APO-M-. I. sRNA-TaBac | Home [Internet]. [cited 11 Apr 2017]. Available: http://srnatabac.toulouse.inra.fr:8080/.

78. Kerpedjiev P, Hammer S, Hofacker IL. Forna (force-directed RNA): simple and effective online RNA secondary structure diagrams. Bioinformatics. 2015;31:3377-9.

\section{Submit your next manuscript to BioMed Central and we will help you at every step:}

- We accept pre-submission inquiries

- Our selector tool helps you to find the most relevant journal

- We provide round the clock customer support

- Convenient online submission

- Thorough peer review

- Inclusion in PubMed and all major indexing services

- Maximum visibility for your research

Submit your manuscript at www.biomedcentral.com/submit
Biomed Central 